\title{
AGOTAMIENTO LABORAL EN PROFESIONALES DE SALUD DE LIMA METROPOLITANA
}

\author{
Alejandro loli P. ${ }^{1}$, Fernando Arias Galicia, Jorge Alarcón V., Rudi Loli P. \\ Universidad Nacional Mayor de San Marcos, Lima, Perú \\ (RECIBIDO EL 21/10/2008, ACEPTADO EL 11/12/2008)
}

\begin{abstract}
RESUMEN
La presente investigación tuvo como objetivo conocer el grado de agotamiento laboral en los profesionales de salud que laboran en los centros de salud de Lima Metropolitana, en una muestra de 89 sujetos que cursaban estudios de posgrado en diversos centros superiores de estudio. El estudio muestra que el agotamiento laboral en los profesionales de salud es normal con tendencia a ser aún más bajo. Una exploración más minuciosa entre los componentes permite encontrar un conjunto de correlaciones, entre los cuales tenemos que existe una asociación negativa entre el estrés y la satisfacción con el salario, la relación con los pacientes, el medio ambiente físico y la salud. Igualmente, asimismo hay asociación negativa entre vida familiar y estrés, satisfacción con el trabajo y estrés, horario y estrés, satisfacción con la vida y estrés, y suficiencia de materiales y estrés. Asimismo existe una relación positiva entre desgaste emocional y estrés, despersonalización y estrés, y logro y estrés.

Finalmente, un análisis con las variables demográficas nos indica que existe asociación entre satisfacción con el trabajo y sexo, y tipo de contrato; vida familiar con edad, tipo de contrato, y trabajar en otra institución; despersonalización con nivel de estudios alcanzados; realización profesional con tiempo de trabajo; compromiso afectivo con antigüedad en la institución y con ingreso neto mensual; estrés con ingreso neto mensual; y satisfacción salarial con ingreso neto mensual.
\end{abstract}

Palabras clave: Agotamiento laboral, estrés, profesionales de salud, Perú.

\begin{abstract}
The present investigation had as objective to know the level of work exhaustion in health professionals that work in health services of metropolitan area of Lima, in a sample of 89 subjects who were postgraduate students in universities of Lima. The study shows that the work exhaustion in the health professionals is normal with tendency to be still lower. Specifically, we found a negative correlation between stress and the satisfaction with salary, the relation with the patients, the physical environment, and the health state. Also, there was a negative correlation between stress and familiar life, work satisfaction, work schedule, life satisfaction, and enough supplies to work. On the other hand, we found a positive correlation between stress and emotional wearing, depersonalization, and success.
\end{abstract}

1 Docente Principal y Ex Decaño de la Facultad de Psicología de la UNMSM, Lima, Perú. E-mail: alolip@unmsm.edu.p 
Finally, an analysis with the demographic variables indicates to us that it exists association between work satisfaction with sex and type of contract; family life with age, type of contract, and other employ; depersonalisation with academic level; professional accomplishment with working time; affective commitment with antiquity in the institution and monthly net salary; stress with monthly net salary; and economic satisfaction with monthly net salary.

Keywords: Work exhaustion, stress, health professional, Peru.

\section{INTRODUCCIÓN}

El término agotamiento o desgaste profesional, denominado también "síndrome de burnout", fue utilizado por primera vez por el psicólogo clínico Herbert Freudenberger (1974) para definir el desgaste extremo de un empleado. Sin duda, en las últimas décadas, algunos estudiosos le denominaron como "quemado", "extenuado", "fundido", "desmotivado" y también como "chamuscado", "oxidado" o "destruido" a estados de salud similares; aunque otros estudiosos encuentran diferencias entre ellos, no están muy lejos de la realidad.

Actualmente este síndrome es una de las causas más importantes de incapacidad laboral en profesionales de la salud; no obstante, el estrés se puede presentar en cualquier tipo de trabajo y debe entenderse como reacciones físicas y emocionales nocivas que ocurren cuando las exigencias del trabajo sobrepasan las capacidades, los recursos o las necesidades del trabajador. El estrés ocasionado por el trabajo puede llevar hasta quebrantar la salud y deteriorar la misma si los estresores permanecen en el tiempo.

Las condiciones del trabajo, las exigencias excesivas del mismo, las expectativas del empleador, el clima organizacional y las propias características del profesional y su capacidad de ajuste a las situaciones estresantes, han contribuido a que el síndrome de burnout afecte cada vez a un mayor número de profesionales. Si a esta situación se le añade las presiones de los pacientes, de la familia y del ambiente social, político y económico, se tendrá mayores condicionantes que afecten a los profesionales. Las investigaciones de Bronfman, Castro, Zúñiga, Miranda, y Oviedo (1997), confirman este punto de vista; ellos entrevistaron a 61 prestadores de servicios de salubridad en cinco regiones de México (excluyendo al D. F.) y encontraron que los médicos laboran en más de una institución (por falta de recursos humaños, salarios bajos y estímulos casi inexistentes); llenan muchos formatos, tienen supervisión administrativa estrecha, y supervisión médica casi inexistente; el equipo es deficiente, obsoleto y carente de mantenimiento adecuado; se presenta insuficiencia en el abasto de medicamentos; faltan espacios apropiados y el mantenimiento de las instalaciones es deficiente. Además, deben atender a muchos pacientes y no pueden dedicar suficiente tiempo a cada uno.

Según Maslach, Schaufeli y Leiter (2001: 397), el "síndrome de burnout” es una respuesta prolongada ante estresores emotivos e interpersonales crónicos en el trabajo, y es definido por tres dimensiones: postración, despersonalización e ineficacia”. La primera dimensión constituye el síntoma central más obvio y está relacionado con el estrés, se refiere al sentimiento de haber agotado los recursos emocionales y físicos personales; la segunda se entiende como una respuesta negativa, endurecida o indiferente en extremo ante varias facetas del trabajo, con referencia especial a las relaciones interpersonales, implica una 
deshumanización; la tercera dimensión (evaluadora respecto al trabajo), significa un sentimiento de incompetencia y una falta de logros y productividad en las labores (Maslach, Schaufeli y Leiter, 2001: 398). Se piensa que las dos primeras facetas conducen a la tercera. Puede verse, entonces, la complejidad del síndrome del agotamiento o desgaste profesional.

Se ha encontrado también que uno de los factores con mayor incidencia sobre la producción del estrés es la imposibilidad de tener control sobre los sucesos y, más específicamente, sobre los acontecimientos e incidentes sobre la propia persona (Theorell y Karasek, 1996). Afecta el desempeño en la forma de ausentismo, intención de renuncia o abandono (Hughes, 2001), en su caso, menor efectividad (Molina Siguero, García Pérez, Alonso González y Cecilia Cermeño, 2003; Wright y Hobfoll, 2004); igualmente, afecta el compromiso con la organización (Wright y Hobfoll, 2004); la salud física (ver, como ejemplo, a Molina Siguero, García Pérez, Alonso González y Cecilia Cermeño, 2003 y otros); la salud mental (Sargent, Sotile, Rubash y Barrack, 2004; Wright y Hobfoll, 2004); las relaciones familiares, entre otras.

Las investigaciones desarrolladas a nivel latinoamericaño y en Perú no son muchas. Liz K. Coronado (2006) aplicó la prueba de Maslach a 42 enfermeros asistenciales de los servicios de medicina y áreas críticas de un hospital de servicio público de Perú, encontrando que la mayoría de las enfermeras tiene un nivel de estrés medio, en relación a la dimensión de agotamiento emocional, despersonalización y realización personal. En cuanto al nivel de estrés laboral, provocados por presión y exigencia organizativa y ambiental, presentan un nivel medio. Los principales factores que ocasionan estrés laboral, según el estudio, son la escasez de personal, mayor demanda de atención, personal con escasa experiencia, el sentirse evaluada, supervisión autoritaria, instrucciones contradictorias, sobrecarga de trabajo, falta de disponibilidad de los equipos, entre otros.

Estudios similares fueron desarrollados por Giovanna Mercado Torres (2006), e Hilda Dávalos Pérez (2005), quienes utilizaron el mismo inventario de Maslach en dos centros de salud diferentes de Perú, y con muestras igualmente diferentes. En el primer estudio se llegó a la conclusión de que el nivel de estrés laboral, en la mayoría de los sujetos participantes, era de nivel medio. Respecto de las dimensiones, en el primer estudio refieren sentirse afectados en la dimensión realización personal, dado que las dimensiones de cansancio emocional y despersonalización no significan un problema. Y, en el segundo estudio, la dimensión agotamiento emocional y despersonalización presentan un nivel medio de estrés, pero la dimensión realización personal es la más afectada, al igual que en el estudio anterior.

Harrison-BA-Hons (2000) entrevistó exhaustivamente a 99 mujeres médicos en cinco estados de México; además, les solicitó que llevaran un diario, durante tres meses, sobre sus experiencias cotidianas. Sus principales hallazgos estriban en los conflictos y tensiones entre los diversos roles que desempeñan (profesional, líder, colega, madre, pareja, hija, empleadora de trabajadoras domésticas). No obstante, dejó fuera el agotamiento profesional.

Jorge Román Hernández (2003) realizó un estudio transversal acerca de estrés y Burnout a 287 profesionales (médicos y enfermeros) de la salud de un municipio de La Habana, 
hombres y mujeres, a través de tres cuestionarios: Cuestionario Breve de Burnout, de Moreno. Escala de Síntomas de Estrés, de Aro; e Inventario de Estrés para Profesionales de la Salud, de Wolfgang. Se encontró que tanto el género y la profesión como el nivel de atención también diferenciaban significativamente las respuestas de estrés y Burnout. Igualmente, el bajo reconocimiento profesional se asociaba a respuestas de estrés y de Burnout. La prevalencia de Burnout fue ligeramente superior a $30 \%$ y la de síntomas de estrés de 50\%. El Burnout afectó fundamentalmente a las mujeres médicas de atención primaria, y el estrés a las enfermeras del mismo nivel de atención.

G. Blanco (2004) desarrolló un estudio sobre el estrés laboral y salud en enfermeras instrumentistas, considerando la distribución de responsabilidades en el hogar, y la salud percibida en una muestra de 54 enfermeras que laboran en cinco centros asistenciales del área Metropolitana de Caracas, con una edad promedio de 37 años. Las variables fueron evaluadas a través de medidas de autorreporte e incluyeron: estrés laboral (demanda-control y apoyo social), distribución de responsabilidades en el hogar, salud física y mental. Los resultados indican que los participantes percibieron altas demandas laborales, pero al mismo tiempo una mayor capacidad de decisión y apoyo social. En la distribución de responsabilidades en el hogar, se observó una mayor participación de estas mujeres en la planificación y gerencia de las tareas, sin embargo, en cuanto a la realización de las mismas recibían ayuda de otros familiares, incluyendo la pareja y ayuda externa. Las altas demandas laborales se asociaron con un mayor reporte de síntomas, así como la percepción de la capacidad de decisión derivan la presencia de una mayor autoestima, menores niveles de depresión y menor porcentaje de síntomas; en cuanto al apoyo social se asoció con un menor reporte de síntomas.

M. A. Pérez San Gregorio, A. Martín Rodríguez, A. Gallego Corpa, E. Correa Chamorro, J. Pérez Bernal (2003), investigaron sobre las repercusiones psicológicas del estrés laboral en 18 enfermeros que trabajaban en los equipos de trasplante de un hospital universitario de Sevilla, los mismos que fueron divididos en dos subgrupos en función de sus niveles (bajo y alto) de depresión y/o ansiedad. Los resultados indicaron que la fuente de estrés que más tensión genera en el personal de enfermería es el "contacto con el dolor y la muerte", los profesionales con mayores niveles de depresión y/o ansiedad son los que más tensión experimentan ante situaciones de "dolor y muerte" y los "conflictos con los pacientes y sus familiares", existiendo una correlación positiva entre el estrés laboral y la sintomatología depresiva y/o ansiosa.

Como se ve, emprender investigaciones más detalladas respecto al agotamiento profesional en el personal de salud y en otras actividades del quehacer laboral es cada vez más necesario. Por ejemplo, una interrogante, aún no resuelta en el ámbito internacional, es el peso específico de los factores personales, sociales y organizacionales en la incidencia del desgaste profesional.

Por estas razones nos interesó conocer los niveles de agotamiento laboral y su relación con las dimensiones: postración, despersonalización e ineficacia. Igualmente, la relación con datos demográficos (sexo, edad, experiencia, etc.) y otros segmentos organizacionales. 


\section{MÉTODO}

\section{Muestra}

La muestra estuvo constituida por 89 profesionales de la salud de Lima metropolitana que siguieron cursos de maestría en diversas universidades, de los cuales 75 (84,3\%) eran de sexo masculino y en su mayoría enfermeros. Se esperaba tener muestras más diversas y amplias de profesiones de la salud, por ejemplo: médicos, tecnólogos médicos, obstetrices, psicólogos, asistentes sociales y otros, pero se observó mucha resistencia.

\section{Instrumentos}

El instrumento utilizado fue un cuestionario adaptado y estandarizado por Fernando Arias Galicia (2006), sobre la base del Inventario de Burnout, diseñado por Maslach, Jackson y Leiter (1996), para personal de salud. Esta última herramienta es la más empleada en el ámbito internacional y ha sido ampliamente validada y confiabilizada (véase a Schwarzer, Schmitz y Tang, 2000; Schutte, Toppinen, y Kalimo, 2000; Gil-Monte, 2002; Hwang, Scherer y Fall-Ainina, 2003).

El instrumento adaptado y estandarizado por Fernando Arias Galicia (2006) está conformado por 20 factores: vida familiar, desgaste emocional, logro, despersonalización, satisfacción con el trabajo, compromiso hacia la organización, superior inmediato, horario, satisfacción con la vida, compañeros, suficiencia de materiales, estrés, satisfacción con el salario, relación con los pacientes, medio ambiente físico, realización profesional, búsqueda de otro empleo, salud, y compromiso de conveniencia con la profesión. Haciendo un total de 138 ítemes, todos ellos con siete alternativas de respuesta que van desde totalmente en desacuerdo (1) y totalmente de acuerdo (7). Como se ve no sólo incluye los tres elementos de la propuesta de Maslach, Jackson y Leiter (1996) que definían el estrés, sino propone otros elementos para definir a lo que él llama "agotamiento laboral". Una denominación más amplia que afecta segmentos de la persona y la organización no explorados antes.

En concordancia con el coeficiente alpha de Cronbach, los diferentes factores cumplen con el criterio de estabilidad y confiabilidad, superando el mínimo $(0,20)$ exigido para estos fines. La confiabilidad general lograda en el procesamiento de datos del presente estudio fue de 0,94; por tanto, altamente estable y confiable (Ver Tabla de confiabilidad de componentes).

\section{Procesamiento de datos}

Se utilizó el paquete estadístico SPSS (Statistical Package for the Social Sciences) para el tratamiento estadístico. De manera especial se utilizó el estadístico de confiabilidad Alfa de Cronbach para ver los niveles de confianza de cada uno de los factores, el TestAnderson-Darling para averiguar si hay una aproximación a la distribución normal del puntaje obtenido a partir de los 138 ítemes, y la correlación de Spearman para establecer la asociación entre los diferentes componentes. La prueba Chi cuadrado para el análisis de las variables demográficas fue también de utilidad. 
Por otro lado, con la finalidad de facilitar el tratamiento de los datos, se optó por reducir la escala de siete alternativas de respuesta a tres: Bajo (totalmente en desacuerdo, en desacuerdo), Medio o Normal (casi en desacuerdo, indeciso, casi de acuerdo) y Alto (de acuerdo, totalmente de acuerdo). De manera que, el tratamiento estadístico y los resultados han sido trabajados y descritos dentro de estos parámentros.

\section{RESULTADOS}

\section{Agotamiento de los profesionales de salud}

Una evaluación general del Tabla N. ${ }^{\circ} 1$, sobre el agotamiento de los profesionales de salud que laboran en los diferentes centros de salud de Lima Metropolitana (Perú), a través de 20 factores que conforman el cuestionario, a juzgar por su mediana (4.24), nos muestra que hay una tendencia de los sujetos a mantenerse dentro de los niveles normales.

Tabla N. ${ }^{0} 1$

\begin{tabular}{cccccc}
\hline \multirow{2}{*}{ Promedio } & \multicolumn{2}{c}{ I C*: (95\%) } & \multirow{2}{*}{ Mediana } & \multirow{2}{*}{ Rango } & \multirow{2}{*}{ Varianza } \\
\cline { 2 - 3 } & Límite Inf. & Límite Sup. & & 1,9 & 0,14 \\
\hline 4,254 & 4,175 & 4,333 & 4.239 & 14 \\
\hline
\end{tabular}

$\left(^{*}\right)$ I C: Intervalo de confianza

Los resultados del cuadro anterior se confirman en el Tabla N. ${ }^{\circ}$, donde se observa que el 55,1\% (IC $=44,1,65,6$ ) de sujetos alcanza el nivel medio de agotamiento, el 43,8\% (IC $=33,3,54,8)$ un nivel bajo y, sólo el $1,1 \%(\mathrm{IC}=0,03,6,10)$ se encuentra en el nivel alto de agotamiento; cuyos Intervalos de Confianza (IC) son de 95,00\%.

Tabla N. ${ }^{\circ} 2$

\begin{tabular}{cccc}
\hline Agotamiento & Porcentaje & \multicolumn{3}{c}{ IC $* \mathbf{9 5 \%}$} \\
\hline Bajo & 43,8 & 33,3 & 54,8 \\
Medio & 55,1 & 44,1 & 65,6 \\
Alto & 1,1 & 0,03 & 6,10 \\
\hline
\end{tabular}

$\left(^{*}\right)$ IC: Intervalo de confianza

\section{Relación entre los factores de agotamiento profesional en salud}

En la Tabla N. ${ }^{\circ} 3$ se encuentra el análisis de correlación de los factores del agotamiento laboral de los profesionales de salud. Nos muestra que existe una correlación significativa y positiva entre la Vida Familiar y la satisfacción con el trabajo $(\mathrm{r}=.27 * *)$, compromiso hacia la organización $(\mathrm{r}=.28 * *)$, superior inmediato $(\mathrm{r}=.22 * *)$, horario de trabajo $(\mathrm{r}=.57 * *)$, satisfacción con la vida $\left(\mathrm{r}=.44^{* *}\right)$, suficiencia de materiales $(\mathrm{r}=.22 * *)$, 
satisfacción con el salario $\left(\mathrm{r}=.18^{*}\right)$, relación con los pacientes $\left(\mathrm{r}=.35^{* *}\right)$, realización profesional $\left(\mathrm{r}=.26^{* *}\right)$, postración $(\mathrm{r}=.29 *)$, salud $\left(\mathrm{r}=.42^{* *}\right)$; es decir, cuanto más intensa y satisfactoria es la Vida Familiar mayor es la satisfacción con el trabajo, el compromiso hacia la organización, la relación con el superior inmediato, con el horario de trabajo, la satisfacción con la vida, con los materiales de trabajo, la satisfacción con el salario, la relación con los pacientes, la realización profesional, postración y la salud. Igualmente, existe correlación significativa pero negativa entre la Vida Familiar y el desgaste emocional $\left(\mathrm{r}=-0.47^{* *}\right)$, la despersonalización $\left(\mathrm{r}=-0.41^{* *}\right)$, y el estrés $\left(\mathrm{r}=-0.48^{* *}\right)$; es decir, cuanto más intensa y satisfactoria es la Vida Familiar, menor es el desgaste emocional, la despersonalización y el estrés.

Por otro lado, la misma tabla nos muestra la existencia de una correlación significativa y positiva entre el Desgaste Emocional y la despersonalización $\left(\mathrm{r}=.44^{* *}\right)$, el logro $\left(\mathrm{r}=.27^{* *}\right)$, el estrés $\left(\mathrm{r}=.61^{* *}\right)$ y búsqueda de otro empleo $\left(\mathrm{r}=.27^{* *}\right)$; de manera que, cuanto mayor es el desgaste emocional mayor es la despersonalización, el logro, el estrés y mayor la búsqueda de otro empleo. También existe una correlación significativa pero negativa entre el Desgaste Emocional y la satisfacción con el trabajo $\left(\mathrm{r}=-.33^{* *}\right)$, el compromiso hacia la organización $\left(\mathrm{r}=-.40^{* *}\right)$, horario $\left(\mathrm{r}=-.59^{* *}\right)$, la satisfacción con la vida $\left(\mathrm{r}=-.38^{* *}\right)$, la relación con los compañeros $(\mathrm{r}=-.19 *)$, satisfacción con el salario $(\mathrm{r}=-.24 * *)$, relación con los pacientes $\left(\mathrm{r}=-.30^{* *}\right)$, medio ambiente físico $\left(\mathrm{r}=-.22^{* *}\right)$, realización profesional $\left(\mathrm{r}=-.35^{* *}\right)$, salud $\left(\mathrm{r}=-.46^{* *}\right)$ y postración $\left(\mathrm{r}=-.36^{* *}\right)$; es decir, a mayor desgaste emocional menor satisfacción con el trabajo, menor compromiso hacia la organización, menor satisfacción con el horario, menor satisfacción con la vida, con los compañeros, con el salario, menor relación con los pacientes, menor satisfacción con el ambiente físico, menor realización profesional, menor satisfacción de la salud y menor interés por la postración.

Así mismo, se encuentra la correlación significativa y positiva entre Despersonalización y logro $\left(r=.31^{* *}\right)$, y estrés $(r=.23 * *)$; es decir, a mayor despersonalización mayor logro y mayor estrés. Hay también relación significativa pero negativa entre Despersonalización y satisfacción con el trabajo $\left(\mathrm{r}=-.23^{* *}\right)$, horario $\left(\mathrm{r}=-44^{* *}\right)$, satisfacción con la vida $(\mathrm{r}=-.31 * *)$, relación con los pacientes $(\mathrm{r}=-.47 * *)$, y salud $\left(\mathrm{r}=-.48^{* *}\right)$, lo que significa que a mayor Despersonalización menor satisfacción con el trabajo, con el horario, con la vida, con los pacientes, y menores condiciones de salud.

Se encuentra también una relación significativa y positiva entre el Logro y el estrés $\left(\mathrm{r}=.21^{*}\right)$; es decir, a mayor logro profesional mayor estrés; mientras que hay una relación significativa pero negativa entre Logro y satisfacción con el trabajo $\left(\mathrm{r}=-.45^{* *}\right)$, compromiso hacia la organización $\left(\mathrm{r}=-.32^{* *}\right)$, horario $(\mathrm{r}=-.21 *)$, satisfacción con la vida $(\mathrm{r}=-.40 * *)$, compañeros $(\mathrm{r}=-.24 * *)$, relación con los pacientes $(\mathrm{r}=-.39 * *)$, realización profesional $(\mathrm{r}=-.32 * *)$, compromiso afectivo con la profesión $(\mathrm{r}=-.24 * *)$, salud $(\mathrm{r}=$ $\left.-.39^{* *}\right)$, y compromiso de conveniencia con la profesión $\left(\mathrm{r}=-.24^{* *}\right)$. Vale decir, a mayor logro menor satisfacción con el trabajo, menor compromiso hacia la organización, menor satisfacción con el horario, menor satisfacción con la vida, menor interacción con los compañeros, menor relación con los pacientes, menor realización profesional, menor compromiso afectivo con la profesión, menor condición de salud, y menor compromiso de conveniencia con la profesión. 
Así mismo, existe correlación significativa y positiva entre Satisfacción con el Trabajo y compromiso hacia la organización $(\mathrm{r}=.59 * *)$, con el superior inmediato $\left(\mathrm{r}=.46^{* *}\right)$, con el horario $\left(\mathrm{r}=.38^{* *}\right)$, satisfacción con la vida $\left(\mathrm{r}=.44^{* *}\right)$, con los compañeros $(\mathrm{r}=$ $.52 * *)$, con la cantidad de materiales $(\mathrm{r}=.22 * *)$, satisfacción con el salario $(\mathrm{r}=.32 * *)$, relación con los pacientes $\left(\mathrm{r}=.32^{* *}\right)$, con el medio ambiente físico $\left(\mathrm{r}=.31^{* *}\right)$, con la realización profesional $\left(\mathrm{r}=.46^{* *}\right)$, con el compromiso afectivo con la profesión $(\mathrm{r}=$ $.50 * *)$, con la salud $(\mathrm{r}=.37 * *)$, con la postración $\left(\mathrm{r}=.51^{* *}\right)$ y con el compromiso de conveniencia con la profesión $(\mathrm{r}=.50 * *)$. Es decir, a mayor satisfacción con el trabajo mayor compromiso hacia la organización, con el superior inmediato, con el horario, mayor satisfacción con la vida, con los compañeros, con la cantidad de materiales, mayor satisfacción con el salario, mayor relación con los pacientes, con el medio ambiente físico, con la realización profesional, mayor compromiso afectivo con la profesión, mejores condiciones de salud y postración, y mayor compromiso de conveniencia con la profesión. Sin embargo, encontramos también relación significativa pero negativa entre la Satisfacción con el Trabajo y el estrés $(\mathrm{r}=-.22 * *)$, y la búsqueda de otro empleo $(\mathrm{r}=$ $-.22 * *)$; lo que significa que a mayor satisfacción con el trabajo menor estrés y menor interés por buscar otro empleo.

En la misma tabla se ve que existe relación significativa y positiva entre el Compromiso hacia la Organización y el superior inmediato $\left(\mathrm{r}=.36^{* *}\right)$, el horario de trabajo $(\mathrm{r}=$ $.39 * *)$, la satisfacción con la vida $(\mathrm{r}=.40 * *)$, los compañeros de trabajo $(\mathrm{r}=.29 * *)$, suficiencia de materiales $(\mathrm{r}=.32 * *)$, satisfacción con el salario $(\mathrm{r}=.31 * *)$, relación con los pacientes $(\mathrm{r}=.24 * *)$, medio ambiente físico $\left(\mathrm{r}=.21^{*}\right)$, realización profesional $(\mathrm{r}=$ $\left..47^{* *}\right)$, compromiso afectivo con la profesión $\left(\mathrm{r}=.35^{* *}\right)$, postración $\left(\mathrm{r}=.51^{* *}\right)$, salud $(\mathrm{r}=$ $.32 * *)$, y compromiso de conveniencia con la profesión $\left(\mathrm{r}=.35^{* *}\right)$; lo que significa que a mayor compromiso hacia la organización, mayor compromiso con el superior inmediato, con el horario de trabajo, la satisfacción con la vida, con los compañeros de trabajo, con la cantidad de materiales, satisfacción con el salario, mayor relación con los pacientes, mayor satisfacción con el medio ambiente físico, mayor realización profesional, compromiso afectivo con la profesión, salud, mayor postración y compromiso de conveniencia con la profesión. Sin embargo, encontramos también una relación significativa pero negativa entre el Compromiso hacia la Organización y la búsqueda de otro empleo $\left(\mathrm{r}=-.41^{* *}\right)$, es decir, que a mayor compromiso hacia la organización menor el interés por buscar otro empleo.

Igualmente, existe correlación significativa y positiva entre el Superior Inmediato y el horario de trabajo $\left(\mathrm{r}=.20^{*}\right)$, los compañeros $\left(\mathrm{r}=.27^{* *}\right)$, la suficiencia de materiales $(\mathrm{r}=.24 * *)$, medio ambiente físico $(\mathrm{r}=.23 * *)$, compromiso afectivo con la profesión $(\mathrm{r}=22 * *)$, postración $(\mathrm{r}=.41 * *)$ y compromiso de conveniencia con la profesión $(\mathrm{r}=.22 * *)$; es decir, a mayor ingerencia del superior inmediato mayor aceptación del horario de trabajo, mejor relación con los compañeros, mayor aceptación de la suficiencia de materiales, del ambiente físico, del compromiso afectivo con la profesión, mayor postración y compromiso de conveniencia con la profesión.

También hay una relación significativa y positiva entre el Horario de trabajo y la satisfacción con la vida $\left(\mathrm{r}=.41^{* *}\right)$, suficiencia de materiales $(\mathrm{r}=.23 * *)$, satisfacción con el salario $\left(\mathrm{r}=.31^{* *}\right)$, relación con los pacientes $(\mathrm{r}=.28 * *)$, medio ambiente físico 
$(\mathrm{r}=.20 *)$, realización profesional $(\mathrm{r}=.39 * *)$, salud $(\mathrm{r}=.42 * *)$ y postración $(\mathrm{r}=.42 * *)$; lo que quiere decir que a mayor aceptación del horario de trabajo mayor satisfacción con la vida, mayor aceptación a la suficiencia de materiales, satisfacción con el salario, mayor relación con los pacientes, con el medio ambiente físico, mayor realización profesional, mayor preocupación por la postración y la salud. Por otro lado hay un relación altamente significativa pero negativa entre el Horario de trabajo y el estrés $\left(\mathrm{r}=-.41^{* *}\right)$; vale decir, que a mayor aceptación o mejor horario de trabajo menor estrés.

En la misma tabla, encontramos que existe una relación significativa y positiva entre Satisfacción con la Vida y los compañeros $(\mathrm{r}=.19 *)$, suficiencia de materiales $(\mathrm{r}=.20 *)$, relación con los pacientes $\left(\mathrm{r}=.45^{* *}\right)$, realización profesional $\left(\mathrm{r}=.38^{* *}\right)$, compromiso afectivo con la profesión $\left(\mathrm{r}=.29^{* *}\right)$, salud $\left(\mathrm{r}=.47^{* *}\right)$, postración $(\mathrm{r}=.19 . *)$ y compromiso de conveniencia con la profesión $\left(r=.29^{* *}\right)$; es decir, a mayor satisfacción con la vida mayor aceptación a los compañeros, a la suficiencia de materiales, mayor relación con los pacientes, mayor realización profesional, compromiso afectivo con la profesión, mejores condiciones de salud y postración, y mayor compromiso de conveniencia con la profesión. Se encuentra también una correlación significativa pero negativa entre Satisfacción con la vida y estrés $(\mathrm{r}=-.32 * *)$ y búsqueda de otro empleo $(\mathrm{r}=-.29 * *)$; es decir, a mayor satisfacción con la vida menor estrés y menor interés por buscar otro empleo.

Por otro lado, se encuentra una relación significativa y positiva entre Compañeros de trabajo y el medio ambiente físico $\left(\mathrm{r}=.23^{* *}\right)$, la realización profesional $\left(\mathrm{r}=.46^{* *}\right)$, el compromiso afectivo con la profesión $\left(\mathrm{r}=.22^{* *}\right)$, postración $\left(\mathrm{r}=.22^{* *}\right)$ y el compromiso de conveniencia con la profesión $(\mathrm{r}=.22 * *)$; lo que significa que cuanto mayor es la aceptación a los compañeros de trabajo mejora el medio ambiente físico, mayor realización profesional, compromiso afectivo con la profesión, mayor interés por la postración y mayor compromiso de conveniencia con la profesión.

De la misma manera encontramos una relación significativa y positiva entre la Suficiencia de Materiales y la satisfacción con el salario $\left(\mathrm{r}=.25^{* *}\right)$ y postración $\left(\mathrm{r}=.22^{* *}\right)$; vale decir, a mayor suficiencia de materiales mayor satisfacción con el salario y mayor preocupación por la postración. Hay también una correlación significativa pero negativa entre la suficiencia de materiales y el estrés $(r=-.32 * *)$; lo que significa que a mayor suficiencia de materiales menor estrés.

También se puede apreciar la existencia de una relación significativa pero negativa entre Estrés y satisfacción con el salario $\left(\mathrm{r}=-.22^{* *}\right)$, relación con los pacientes $\left(\mathrm{r}=-.27^{* *}\right)$, ambiente físico $\left(\mathrm{r}=-.25^{* *}\right)$, y salud $\left(\mathrm{r}=-.25^{* *}\right)$. Vale decir, que a mayor estrés menor satisfacción con el salario, menor relación con los pacientes, menor aceptación del ambiente físico y menores condiciones de salud.

Igualmente, se encuentra una relación significativa y positiva entre Satisfacción con el salario y postración $\left(\mathrm{r}=.35^{* *}\right)$, es decir, a mayor satisfacción con el salario mayor preocupación por la postración; surgiendo también una relación significativa pero negativa entre Satisfacción con el salario y búsqueda de otro empleo $(\mathrm{r}=-.29 * *)$, lo que quiere decir que a mayor satisfacción con el salario menor interés por buscar un nuevo empleo.

Por otro lado, existe una relación significativa y positiva entre Relación con Pacientes y realización profesional $\left(\mathrm{r}=.39^{* *}\right)$, compromiso afectivo $\left(\mathrm{r}=.29^{* *}\right)$, salud $\left(\mathrm{r}=.46^{* *}\right)$ 
postración $(\mathrm{r}=.25 * *)$, compromiso de conveniencia $(\mathrm{r}=.29 * *)$; lo que significa que cuanto mayor y mejor es la relación con los pacientes mayor es la realización profesional y el compromiso afectivo, mejora la postración y el compromiso de conveniencia. De la misma manera, encontramos una relación significativa pero negativa entre Relación con Pacientes y búsqueda de otro empleo $\left(\mathrm{r}=-.35^{* *}\right)$, es decir, a mayor relación con pacientes menor interés de buscar otro empleo.

Así mismo, encontramos una relación significativa y positiva entre Realización Profesional y compromiso afectivo $\left(\mathrm{r}=.21^{*}\right)$, salud $\left(\mathrm{r}=.34^{* *}\right)$, y compromiso de conveniencia $\left(\mathrm{r}=.21^{*}\right)$, es decir, a mayor realización profesional, mayor compromiso afectivo, salud y compromiso de conveniencia.

Igualmente, se encuentra una relación significativa y negativa entre Búsqueda de otro Empleo y salud $\left(\mathrm{r}=-.31^{* *}\right)$, postración $\left(\mathrm{r}=.23^{* *}\right)$; es decir, que cuanto mayor es el interés por buscar otro empleo, menor es la preocupación por la postración y la salud.

Del mismo modo, encontramos una relación significativa y positiva entre Salud y postración $(\mathrm{r}=.27 * *)$ y compromiso de conveniencia $(\mathrm{r}=.29 * *)$; significando que, cuanto mejor es la condición de salud mejora la postración y mayor es el compromiso de conveniencia.

Encontramos también relación significativa y positiva entre Postración y compromiso de conveniencia $\left(r=.23^{*}\right)$, lo que significa que cuanto mayor es la postración mayor es el compromiso de conveniencia. 


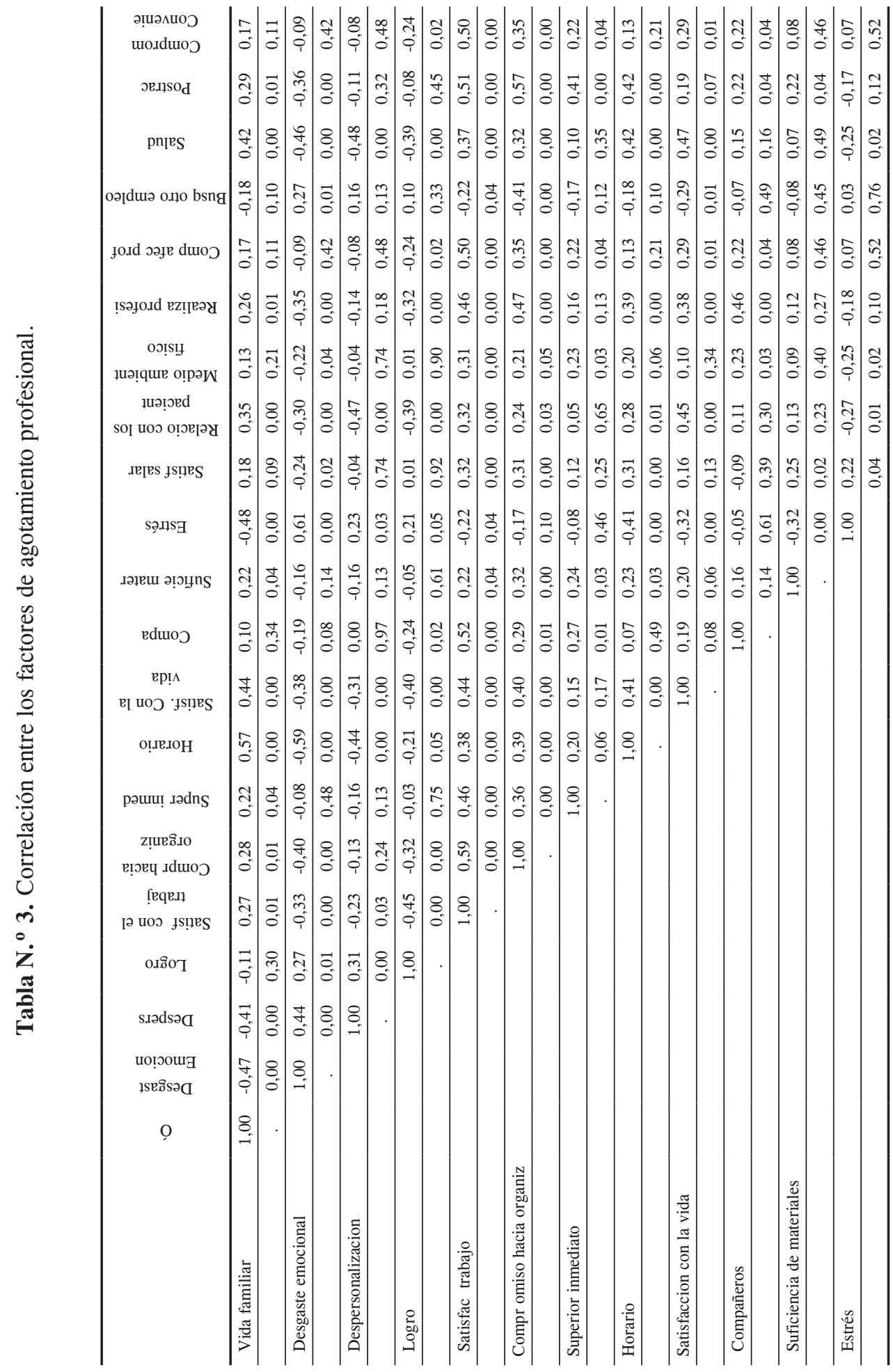




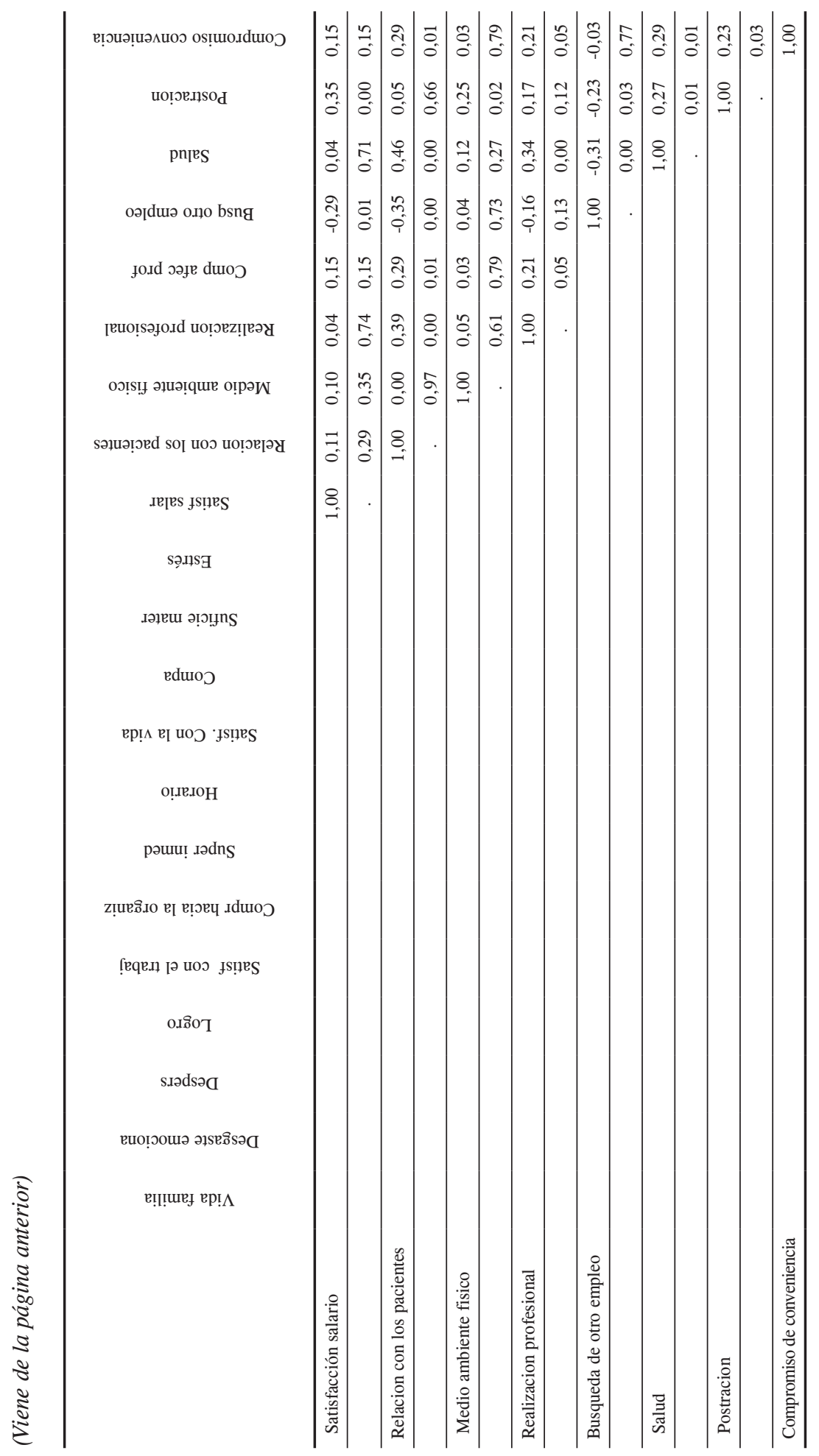




\section{Agotamiento profesional en salud según variables demográficas}

Las tablas que siguen describen la asociación entre el agotamiento profesional de los sujetos participantes, de acuerdo a la intervención de las variables demográficas que resultaron significativas. La muestra total varía en algunos casos por falta de datos de algún participante, casos en los que se incluirá el número de muestra para una mejor comprensión del análisis.

\section{Satisfacción con el trabajo según sexo}

Como se aprecia en la Tabla N. ${ }^{\circ} 4$ (anexo), de acuerdo con la prueba, razón de verosimilitud y a través de la prueba de Chi Cuadrado, existe una asociación entre la satisfacción con el trabajo y el sexo, con un nivel de confiabilidad del 95\% ( $\mathrm{p}<=0.05)$. En esta relación, aquellas personas que refirieron estar altamente satisfechos con el trabajo, el 94,6 \% fueron mujer y los hombres solo un $5,4 \%$. Igualmente, los que contestaron estar normalmente satisfechos el 76,5\% fueron mujeres y el 23,5\% fueron varones. Probablemente, se debe a que las mujeres son más concientes y realistas sobre la escasez de trabajo, de manera que incluso las condiciones de trabajo son mejor aceptados que por los varones.

\section{Satisfacción con el trabajo según el tipo de contrato}

La Tabla N. ${ }^{\circ} 5$ (anexo) analiza la satisfacción con el trabajo de los profesionales de salud en relación con el tipo de contrato que mantiene con la organización. Siguiendo el mismo procedimiento del análisis anterior, en una muestra total de 88 sujetos, se encontró una asociación entre la satisfacción con el trabajo y el tipo de contrato, con un nivel de confiabilidad del $90 \%(\mathrm{p}<=0.10)$. En esta relación, aquellas personas que refirieron estar medianamente satisfechos con el trabajo, el 54,9\% eran profesionales nombrados, siendo esta relación más alta que los de contratos eventuales y los de contratos por servicios no personales. En cambio, aquellas personas que refirieron estar altamente satisfechos con el trabajo, el 50,0\% tenía contrato por servicios no personales, los nombrados solo un $38,9 \%$ y los que tenían contratos eventuales sólo el 11,1\%. Esto se debe, sin duda, a que las personas que tienen contrato por servicios no personales cuidan y aprecian más su trabajo probablemente por la inestabilidad laboral de su contrato, mientras que los nombrados a pesar de la estabilidad laboral están menos satisfechos probablemente debido a expectativas incumplidas.

\section{Vida familiar según la edad de los profesionales de la salud}

La Tabla No 6 (anexo) nos muestra el grado de armonía entre la familia y el trabajo de acuerdo a la edad de los profesionales de salud. Aquí, hay una asociación entre la vida familiar y los grupos de edad, con un nivel de confiabilidad del 90\% ( $<<=0.10)$. En esta relación, aquellas personas que refirieron tener una vida familiar armoniosa, buena y satisfactoria (alta) se encontraban entre los 31 y los 40 años de edad (45.9\%), seguido por aquellos que tenían entre 21 y 30 años (26.2\%), disminuyendo a $21.3 \%$ los que tenían 
entre 41 y 50 y a $6.6 \%$ aquellos que tenían más de 51 años de edad. Probablemente, debido a que entre 31 y 40 años de edad los profesionales ya lograron estabilidad económica, social y familiar. Antes de esas edades los jóvenes profesionales tienen relativa estabilidad, porque la mayoría de ellos toman en cuenta a sus padres como su propia familia; entre tanto después de esas edades, la relación vida familiar y trabajo tiende a ser normal (medio: $50,0 \%$ ) por su madurez emocional y profesional junto a su estabilidad económica.

\section{Vida familiar según tipo de contrato de los profesionales de la salud}

En la Tabla N. ${ }^{\circ} 7$ (anexo) se puede apreciar la relación entre vida familiar de los profesionales de salud y el tipo de contrato laboral que tienen en su trabajo. En una muestra total de 88 sujetos, se encontró una asociación entre la vida familiar y los tipos de contrato laboral, con un nivel de confiabilidad del $95 \%(\mathrm{p}<=0.05)$. En esta relación, aquellas personas que refirieron tener una vida familiar armoniosa, buena y satisfactoria (alta) eran los que tenían contrato por servicios no personales $(45,9 \%)$, frente a profesionales nombrados $(44,3 \%)$ y contratos eventuales $(9,0 \%)$. En cambio, los que señalaron tener vida familiar normal (medio) fueron los nombrados $(73,7 \%)$, seguido por los contratados por servicios no personales $(26,3 \%)$. Mientras que los que señalaron que tenían una vida familiar baja, no armoniosa, insatisfecha etc. fueron los que tenían contrato laboral por servicios no personales $(75,0 \%)$. Estas cifras nos pueden estar indicando que hay una inestabilidad laboral muy alta que está afectando la vida familiar, al mismo tiempo que hay un alto porcentaje de profesionales de la salud nombrados que dicen tener una vida familiar normal.

\section{Vida familiar de los profesionales de la salud según trabaja en otra institución}

La Tabla N. ${ }^{o} 8$ (anexo), demuestra que hay una asociación entre vida familiar y trabajo en otra institución, con un nivel de confiabilidad del $90 \%(\mathrm{p}<=0.10)$. En esta relación, aquellas personas que refirieron tener una vida familiar armoniosa, buena y satisfactoria (alta), en un $63.9 \%$ no trabajaba en otra institución y el $36.1 \%$ si trabajaban en otra institución. Sin embargo, aquellos que contestaron que tenían una vida familiar normal, el $55.0 \%$ trabajaba en otra institución y el resto que no trabajaba en otra institución. Por otro lado, los que refirieron tener una vida familiar baja, el $87.5 \%$ no trabajaba en otra institución, el resto sí. Probablemente se debe a que tienen un ingreso económico importante para una vida familiar adecuada y, en otros casos, las restricciones laborales no les permiten trabajar en otra institución y por consiguiente dicen tener una vida familiar más modesta e insatisfactoria.

\section{Despersonalización según nivel de estudios de los profesionales de la salud}

En la Tabla $\mathrm{N}^{\circ} 9$ (anexo), se puede apreciar que existe una asociación entre despersonalización y el nivel de estudios alcanzados, con un nivel de confiabilidad del $90 \%(\mathrm{p}<=0.10)$. En esta relación, aquellas personas que refirieron tener un grado normal (medio) de despersonalización, el $84.6 \%$ eran profesionales con título académico 
y el resto tenía estudios de posgrado. Igualmente, los que indicaron tener un grado bajo de despersonalización, el $67,7 \%$ eran profesionales con título académico y el resto con estudios de posgrado.

\section{Realización profesional según el tiempo de trabajo}

La Tabla N. ${ }^{\circ} 10$ (anexo) nos indica que hay una asociación entre realización profesional y el tiempo de trabajo realizado durante toda su vida, con un nivel de confiabilidad del $95 \%(\mathrm{p}<=0.05)$. En esta relación, aquellas personas que refirieron tener un alto grado de realización profesional habían trabajado entre 11 y 20 años, y los que refirieron tener un grado normal (medio) de realización profesional habían trabajado entre 6 y 10 años; pero ningún profesional refirió tener un grado bajo de realización profesional. Estos resultados pueden ser el reflejo del concepto tradicional que se tiene del profesional de salud, que ha sido culturalmente aceptado como alto en relación a otras profesiones; motivo por el cual estas personas pueden creer que son superiores a las demás que tienen otras profesiones aún cuando pueden ser pocos sus años de ejercicio profesional.

\section{Superior inmediato según antigüedad en la institución de los profesionales de salud}

La Tabla $\mathrm{N}^{\circ} 11$ (anexo), encontramos que hay una asociación entre el superior inmediato y la antigüedad en la institución, con un nivel de confiabilidad del $95 \%(\mathrm{p}<=0.05)$. En esta relación, aquellas personas que refirieron tener un alto concepto y buenas relaciones con su superior inmediato, en su mayoría tenían entre 6 y 10 años de antigüedad en la institución (40,5\%); del mismo modo, quienes dijeron tener una relación normal y aceptable con su superior inmediato, en su mayoría tenía entre 11 y 20 años de antigüedad (35,3\%); entre tanto los que indicaron tener una relación todavía débil, y un concepto bajo, en su mayoría tenían una antigüedad menor a un año (33,3\%). Probablemente por que todas las personas al ingresar a trabajar a una institución sufren un proceso de adaptación, siendo sus relaciones inseguras, temerosas y débiles, mejorando en la medida que pasan los años, y disminuyendo luego en los últimos años de vida laboral.

\section{Superior inmediato según antigüedad en el puesto de trabajo de los profesionales de salud}

La Tabla $\mathrm{N}^{\circ} 12$ (anexo), encontramos, en una muestra total de 88 sujetos, que hay una asociación entre el superior inmediato y la antigüedad en el puesto de trabajo, con un nivel de confiabilidad del $95 \%(\mathrm{p}<=0.05)$. En esta relación aquellas personas que refirieron tener un alto concepto y buenas relaciones con el superior inmediato, en su mayoría tenían entre 1 y 10 años $(80,5 \%)$ de antigüedad en el puesto de trabajo; los que señalaron tener una relación normal con el superior inmediato, en su mayoría tenían entre 1 y 5 años $(38,2 \%)$ de antigüedad en el puesto; mientras que los que indicaron que tenían un concepto bajo del superior inmediato, en su mayoría tenían menos de 1 año $(33,3 \%)$ 
de antigüedad, aunque en los años posteriores se mantiene también bajo el concepto del superior inmediato. Esto parece estar vinculado a los éxitos logrados en la ejecución del puesto y a la riqueza de las tareas.

\section{Compromiso afectivo con la profesión según antigüedad en la institución de los profesionales de salud}

En la Tabla N. ${ }^{\circ} 13$ (anexo), se puede observar que hay una asociación entre el compromiso afectivo con la profesión y la antigüedad en la institución, con un nivel de confiabilidad del $90 \%(p<=0.10)$. En esta relación, aquellas personas que refirieron tener un alto compromiso afectivo con la profesión tenían una antigüedad entre 6 y 10 años $(29,4 \%)$; sin embargo, se puede decir que el compromiso se mantiene por encima de $20,6 \%$ en todos los profesionales desde su ingreso hasta los 20 años de servicios en la institución. Igual ocurre con aquellos que señalaron que tenían un compromiso afectivo normal, siendo el más alto entre 1 y 5 años de antigüedad (28,8\%) y prolongándose hasta los 30 años. Los profesionales que refirieron tener un compromiso afectivo con la profesión bajo fueron pocos y también se mantiene durante todo el tiempo de permanencia en la institución. Probablemente se debe no necesariamente a las influencias de la institución sino básicamente a la formación académica, salvo condiciones laborales similares ofrecidas por las instituciones, pero dada la diversidad de la muestra éste es improbable.

\section{Estrés laboral según ingreso neto mensual de los profesionales de salud}

En la Tabla N. ${ }^{0} 14$ (anexo), apreciamos que hay una asociación entre el estrés laboral y el ingreso neto mensual, con un nivel de confiabilidad del $95 \%(\mathrm{p}<=0.05)$. En esta relación, aquellas personas que refirieron tener un estrés laboral alto ( 8 personas de la muestra: $100,0 \%)$ eran los que tenían un ingreso neto mensual entre 1001 y 3000 soles. Mientras que aquellos que indicaron tener estrés normal (medio), el 51,7\% tenía un ingreso neto mensual hasta 1000 soles, disminuyendo dicho porcentaje en la medida en que aumentaba sus ingresos. Algo similar ocurre con los profesionales que declararon tener un estrés bajo, siendo más significativo en aquellos que tenían entre menos de 1000 hasta 3000 soles de ingreso neto mensual. Estos resultados se deben, sin duda, a los bajos ingresos económicos de los profesionales de la salud en compensación a sus tareas cotidianas, mejorando sus condiciones de estrés a mayor ingreso.

\section{Satisfacción salarial según ingreso neto mensual de los profesionales de salud}

En la Tabla N. ${ }^{\circ} 15$ (anexo), se observa que hay una asociación entre la satisfacción salarial y el ingreso neto mensual, con un nivel de confiabilidad del $90 \%(\mathrm{p}<=0.10)$. En esta relación, aquellas personas que refirieron tener satisfacción salarial alta $(83,3 \%)$ declararon tener un ingreso neto mensual entre 1001 y 3000 soles en relación a los demás. En cambio, aquellos que declararon tener satisfacción salarial normal (medio), tenían un ingreso neto mensual hasta mil soles $(50,0 \%)$, seguido por los que tenían ingreso neto mensual entre 1001 y 3000 soles $(46,7 \%)$. Entre tanto, aquellos que indicaron tener satisfacción salarial 
baja tenían un ingreso neto mensual hasta 1000 soles, seguido por los que tenían ingreso neto mensual entre 1001 y 3000 soles $(42,6 \%)$, en relación al resto que pasaba los 3001 soles y estaba insatisfecho. Esto probablemente se debe a las diferencias en las condiciones de vida profesionales, personales y familiares.

\section{Compromiso afectivo según ingreso neto mensual de los profesionales de salud}

En la Tabla N. ${ }^{\circ} 16$ (anexo), se observa que hay una asociación entre el compromiso afectivo con la profesión y el ingreso neto mensual, con un nivel de confiabilidad del $95 \%$ $(\mathrm{p}<=0.05)$. En esta relación, aquellas personas que refirieron tener un compromiso afectivo con la profesión alto fueron las que tenían un ingreso neto mensual hasta 1000 soles $(52,9 \%)$, disminuyendo los que tenían ingresos entre 1001 y 3000 soles (47,1\%). Mientras que los que señalaron tener compromiso afectivo normal (medio) fueron aquellos que tenían un ingreso neto mensual entre 1001 y 3000 soles $(48,1 \%)$, disminuyendo este porcentaje a 40,4\% con ingresos menores a 1000 soles. Los que declararon compromiso afectivo bajo se concentra entre los que tienen ingreso mensual de 1001 a 3000 soles. Probablemente se debe a que los profesionales de la salud tienen un compromiso afectivo con la profesión muy consistente, que a pesar de los bajos ingresos se mantiene dicho compromiso.

\section{Búsqueda de otro empleo según ingreso neto mensual de los profesionales de salud}

En la Tabla N. ${ }^{\circ} 17$ (anexo), se puede apreciar que hay una asociación entre la búsqueda de otro empleo y el ingreso neto mensual, con un nivel de confiabilidad del $90 \%(\mathrm{p}<=0.10)$. En esta relación, aquellas personas que refirieron tener alto interés por buscar otro empleo tenían un ingreso neto mensual hasta 1000 soles $(45,5 \%)$, en relación a los demás que tenían ingresos mayores. Ocurre de manera similar, en aquellas personas que señalaron su interés normal (medio) de buscar otro empleo, un 52,2\% tenía un ingreso neto mensual hasta 1000 soles, disminuyendo este porcentaje en aquellos que tenían ingresos mayores. Entre tanto, las personas que declararon tener un interés bajo por buscar otro empleo tenían un ingreso neto mensual entre 1001 y 3000 soles $(58,2 \%)$, siendo menor el porcentaje de sujetos $(40,0 \%)$ que tienen ingresos hasta 1000 soles. Estos resultados nos indican que entre los profesionales de la salud no hay mucho interés por buscar otro empleo por razones de escasez, igual se mantiene el interés con ingresos mensuales mayores.

\section{DISCUSIÓN}

A la luz de la teoría y las investigaciones revisadas, los factores condicionantes del agotamiento y específicamente del estrés tienen que ver con las exigencias y responsabilidades del trabajo que van más allá de las capacidades del profesional. Tiene que ver también con las presiones del empleador y otras condiciones del trabajo, con las propias características del profesional y su capacidad de ajuste a las situaciones estresantes, acompañado por las presiones del clima organizacional, las presiones de los pacientes, de la familia y del ambiente social, político y económico. 
Los resultados del presente estudio arrojan, en términos generales, que los profesionales de salud alcanzan el nivel medio de agotamiento e incluso con tendencia a bajar este nivel; en otros términos, la mayoría de los profesionales de salud que forman parte de la muestra no tiene estrés y el $98,9 \%$ de los encuestados podría llegar a tener estrés normal. Lo que significa que los condicionantes del agotamiento y del estrés (Theorell y Karasek, 1996; Hughes, 2001; Liz K. Coronado, 2006) en los centros de trabajo de los integrantes de la muestra parecen no existir o no son suficientes para causar estrés, o los profesionales de salud tienen buena capacidad de ajuste a la diversidad de estresores.

Un análisis más detenido entre los componentes del agotamiento laboral de los profesionales de salud, nos permite afirmar que existe asociación significativa y positiva entre la Vida Familiar y la satisfacción con el trabajo, el compromiso hacia la organización, con el superior inmediato, el horario de trabajo, la satisfacción con la vida, suficiencia de materiales, satisfacción con el salario, relación con los pacientes, realización profesional y con la salud. Pero también una correlación negativa de la vida familiar con el desgaste emocional, la despersonalización, y el estrés. Lo que significa que la vida familiar afecta de una u otra manera a la organización y sus componentes, incluyendo al propio individuo como señala Wright y Hobfoll (2004), cuando habla de compromiso con la organización, o Hughes (2001) cuando se refiere a la relación con el desempeño.

Igualmente, existe asociación positiva entre el Desgaste Emocional y la despersonalización, el logro, el estrés, y búsqueda de otro empleo, y relación negativa con la satisfacción con el trabajo, el compromiso hacia la organización, el horario, la satisfacción con la vida, la relación con los compañeros, la satisfacción con el salario, la relación con los pacientes, el medio ambiente físico, la realización profesional y la búsqueda de otro empleo. Resultado coherente con los hallazgos de Wright y Hobfoll (2004), Sargent, Sotile, Rubash y Barrack (2004), en el sentido de cómo el desgaste emocional puede afectar al indivíduo, su efectividad en la organización y sus relaciones interpersonales e incluso su medio ambiente físico. Ocurre lo mismo con la Despersonalización como afirma Maslach, Schaufeli y Leiter (2001), que afecta la salud mental y física, surgiendo, por tanto, su incompetencia frente a las exigencias organizacionales.

La asociación positiva entre Logro y estrés nos indica que a mayor logro profesional se da mayor estrés, hallazgo, si no contrario, diferente al encontrado por J. Román Hernandez (2003) en una muestra de 287 médicos y enfermeros en La Habana-Cuba, donde el bajo reconocimiento profesional se asocia a respuestas de estrés. Igualmente, el logro profesional en el presente estudio se asocia negativamente con satisfacción con el trabajo, compromiso hacia la organización, horario de trabajo, satisfacción con la vida, con compañeros, relación con los pacientes, con la realización profesional, con el compromiso afectivo con la profesión, con la salud, y con el compromiso de conveniencia con la profesión, debido probablemente al surgimiento de nuevas expectativas.

Por otro lado, la satisfacción con el trabajo se asocia positivamente con el compromiso hacia la organización y con los demás componentes del agotamiento laboral, excepto la asociación negativa con el estrés y la búsqueda de otro empleo; indicándonos que una mayor satisfacción disminuye las posibilidades del estrés y naturalmente el interés por buscar otro empleo. 
Theorell y Karasek (1996) encontraron que uno de los factores con mayor incidencia sobre la producción del estrés es la imposibilidad de tener control sobre los acontecimientos e incidentes sobre la propia persona, situación que afecta el compromiso con la organización (Wright y Hobfoll, 2004), y en la medida en que este último esté asociada con otros elementos organizacionales, tales como el superior inmediato, el horario de trabajo, los compañeros de trabajo, suficiencia de materiales, medio ambiente físico, satisfacción salarial y otras actitudes más personales del profesional, como la relación con pacientes, satisfacción con la vida, realización profesional, compromiso afectivo con la profesión, la salud física y mental, hallados en la presente investigación, nos indica la magnitud de elementos que quedan afectados por los productores del estrés. Es cierto que, en la muestra estudiada, el agotamiento laboral de los profesionales de salud no es un problema, entonces se podría creer que el control sobre los acontecimientos como productores del estrés, por lo menos en parte, está en maños de los profesionales de salud.

De lo anterior se podría deducir que, probablemente, esas son las razones por la que la satisfacción con la vida está asociada positivamente con los compañeros de trabajo, la suficiencia de materiales, la relación con los pacientes, la realización profesional, el compromiso afectivo con la profesión, la salud y el compromiso de conveniencia con la profesión; y por otro lado, la satisfacción con la vida está asociada negativamente con el estrés y con la búsqueda de otro empleo; es decir, a mayor satisfacción con la vida menor estrés y menor interés por buscar otro empleo.

Finalmente, también encontramos una asociación negativa de la suficiencia de materiales con el estrés, del horario de trabajo con el estrés, y, por último, el estrés aparece asociada negativamente con satisfacción con el salario, relación con los pacientes, con el ambiente físico y la salud. Obviamente, el estrés no le permite una interacción armoniosa ni satisfactoria, porque el salario deja de tener el valor justo, se deterioran las relaciones con sus pacientes, el ambiente físico le parece inapropiado y la salud física y mental termina afectada.

Respecto a la relación con algunas variables demográficas, efectivamente se encontró relación entre algunos componentes del agotamiento laboral y dichas variables, como se puede apreciar en las conclusiones.

Todas estas asociaciones nos muestran la coherencia lógica del estudio; sin embargo, una muestra más amplia en el sector podría variar algunos resultados. Naturalmente, un nuevo estudio demandará de una administración del cuestionario en una muestra más variada de profesionales y, si fuera posible, seleccionadas en los propios centros de trabajo.

\section{CONCLUSIONES}

1. El agotamiento de los profesionales de salud que laboran en los diferentes centros de salud de Lima Metropolitana - Perú, a juzgar por su mediana, se encuentra dentro de los niveles normales con tendencia a disminuir a niveles más bajos

2. Una exploración de cada uno de los componentes del agotamiento laboral de los profesionales de salud nos indica que existe confiabilidad de cada uno de los componentes, haciendo consistente la correlación entre ellos. 
3. Existe asociación de algunos componentes del agotamiento laboral con variables demográficas, tales como:

- La satisfacción con el trabajo está asociada con el sexo, siendo más alto el número de mujeres satisfechas con el trabajo que los varones; y con tipo de contrato, siendo más alto el número de contratados por servicios no personales satisfechos con el trabajo que los nombrados.

- Igualmente, el componente vida familiar está asociado a la edad, siendo las personas entre 31 y 40 años las que en mayor porcentaje refieren tener una vida familiar más armoniosa y satisfactoria que otros grupos etáreos; y los contratados por servicios no personales más que los nombrados, y eran los que no trabajaban más que los que si trabajaban en otra institución.

- El componente despersonalización también aparece asociado con el nivel de estudios, siendo los que tienen el título profesional más que los posgraduados los que dicen tener un grado normal o bajo de despersonalización.

- La realización profesional está asociada a tiempo de servicios en el trabajo, donde aquellos que tenían entre 11 y 20 años eran los que señalaban alto grado de realización profesional en relación a los demás.

- El compromiso afectivo con la profesión es otro componente asociado a antigüedad en la institución, donde aquellos que tenían entre 6 y 10 años eran los que decían tener alto compromiso afectivo con la profesión en relación a los demás y normal entre 1 y 5 años; asimismo, este componente aparece asociado a un ingreso neto mensual, siendo aquellos que ganan menos los que dicen tener más alto compromiso afectivo con la profesión.

- El estrés aparece asociado al ingreso neto mensual de los profesionales de salud. Los que respondieron tener bajo nivel de estrés y normal nivel de estrés fueron los que menos ingresos mensuales percibían.

4. La asociación entre los componentes y otras variables demográficas no fueron hallazgos significativos, razón por la cual no se incorpora en el presente artículo.

\section{REFERENCIAS BIBLIOGRÁFICAS}

1. Blanco G. (2004). Estrés laboral y salud en las enfermeras instrumentistas. Revista de la Facultad de Medicina vol. 27 N. ${ }^{\circ} 1$. Caracas.

2. Coronado Luna, L. K. (2006). Factores laborales y niveles de estrés laboral en enfermeros de los servicios de áreas críticas y medicina del Hospital Nacional Daniel A. Carrión. Perú: Universidad Nacional Mayor de San Marcos.

3. Dávalos Pérez, H. Y. (2005). Nivel de estrés laboral en enfermeras del Hospital Nacional de Emergencias José Casimiro Ulloa. Perú: Universidad Nacional Mayor de San Marcos. 
4. Maslach, C., Schaufeli, W.B. y Leiter, M.P. (2001). Job burnout. Annual review of psychology, 52, 397-422.

5. Mercado Torres, G. (2006). Nivel de estrés laboral en enfermeras del Hospital Nacional Dos de Mayo. Perú: Universidad Nacional Mayor de San Marcos.

6. Pérez San Gregorio, M. A; Martín Rodríguez, A; Gallego Corpa, A; Correa Chamorro, A; Pérez Bernal, J. (2003). Repercusiones psicológicas del estrés laboral en los profesionales sanitarios que trabajan en los equipos de trasplantes. Sevilla: Cuadernos de Medicina Psicosomática y Psiquiatría de enlace.

7. Román Hernández, J. (2003). “Estrés y burnout en profesionales de la salud de los niveles primario y secundario de atención”. Rev Cubana Salud Pública. La Habana: Instituto Nacional de Salud de los Trabajadores,

8. The Naty, S. (2007). Sindrome de Burnout. Chile. html.rincondelvago.com/sindromede-burnout.html 


\section{ANEXO DE TABLAS}

Tabla de confiabilidad para cada uno de los componentes

\begin{tabular}{|c|c|}
\hline Componentes (o factores) & Confiabilidad \\
\hline ("Vida familiar") & 0,437 \\
\hline (“Desgaste emocional”) & 0,763 \\
\hline (“Despersonalizacion”) & 0,473 \\
\hline (“Logro") & 0,68 \\
\hline ("Satisfaccion con el trabajo") & 0,781 \\
\hline (“Compromiso hacia la organización”) & 0,74 \\
\hline ("Superior inmediato") & 0,789 \\
\hline ("Horario") & 0,652 \\
\hline (“Satisfaccion con la vida”) & 0,645 \\
\hline (“Compañeros”) & 0,842 \\
\hline ("Suficiencia de materiales") & 0,802 \\
\hline (“Estrés”) & 0,524 \\
\hline (“Satisfacción salario”) & 0,81 \\
\hline ("Relación con los pacientes") & 0,379 \\
\hline (“Medio ambiente físico") & solo tiene una variable \\
\hline (“Realización profesional”) & 0,235 \\
\hline Compromiso afectivo con la profesión & 0,373 \\
\hline (“Compromiso de conveniencia con la profesión”) & 0,297 \\
\hline (“Búsqueda de otro empleo”) & 0,741 \\
\hline (“Salud”) & 0,773 \\
\hline (“Postración”) & 0,814 \\
\hline Confiabilidad general & 0,944 \\
\hline
\end{tabular}


Tabla N. ${ }^{\circ}$ 4. Grado de satisfacción con el trabajo según sexo.

\begin{tabular}{ccccc}
\hline \multirow{2}{*}{ Satisfacción con el trabajo } & \multicolumn{2}{c}{ Sexo } & \multirow{2}{*}{ Total } \\
\cline { 2 - 4 } \cline { 3 - 4 } Bajo & Femenino & Masculino & 0 & 1 \\
\cline { 2 - 3 } \cline { 3 - 4 } Medio & 1 &, $0 \%$ & $100,0 \%$ \\
\hline \multirow{2}{*}{ Alto } & $390 \%$ & 12 & 51 \\
& $76,5 \%$ & $23,5 \%$ & $100,0 \%$ \\
\hline \multirow{2}{*}{ Total } & 35 & 2 & 37 \\
& $94,6 \%$ & $5,4 \%$ & 100,0 \\
\hline
\end{tabular}

$p<=0.05$

Tabla N. ${ }^{0}$ 5. Grado se satisfacción con el trabajo según el tipo de contrato.

\begin{tabular}{ccccc}
\hline Satisfacción con el trabajo & \multicolumn{3}{c}{ Tipo de contrato } & Total \\
\cline { 1 - 4 } Bajo & Contrato SNP & Contrato eventual & Nombrado & \multirow{2}{*}{1} \\
\cline { 2 - 5 } & 0 & 1 & 0 & $100,0 \%$ \\
\cline { 2 - 5 } Medio & 21 & $100.0 \%$ & $0 \%$ & 51 \\
& $41,2 \%$ & 2 & 28 & $100,0 \%$ \\
\hline \multirow{2}{*}{ Alto } & 18 & $4,9 \%$ & $54,9 \%$ & 36 \\
& $50,0 \%$ & $11,1 \%$ & $38,9 \%$ & $100,0 \%$ \\
\hline \multirow{2}{*}{ Total } & 39 & 7 & 42 & 88 \\
& $44,3 \%$ & $8,0 \%$ & $47,7 \%$ & $100,0 \%$ \\
\hline
\end{tabular}

$p<=0.10$

Tabla N. ${ }^{\circ}$ 6. Relación entre vida familiar y la edad.

\begin{tabular}{cccccc}
\hline Vida familiar & \multicolumn{3}{c}{ Edad } & & Total \\
\hline \multirow{2}{*}{ Bajo } & $\mathbf{2 1} \mathbf{y} \mathbf{3 0}$ & $\mathbf{3 1} \mathbf{y} \mathbf{4 0}$ & $\mathbf{4 1} \mathbf{~} \mathbf{5 0}$ & $\mathbf{5 1} \mathbf{y}$ más & \\
\cline { 2 - 5 } & 3 & 4 & 0 & 1 & 8 \\
& $37,5 \%$ & $50,0 \%$ & $0 \%$ & $12,5 \%$ & $100,0 \%$ \\
\hline \multirow{2}{*}{ Medio } & 4 & 4 & 10 & 2 & 20 \\
& $20,0 \%$ & $20,0 \%$ & $50,0 \%$ & $10,0 \%$ & $100,0 \%$ \\
\hline \multirow{2}{*}{ Alto } & 16 & 28 & 13 & 4 & 61 \\
& $26,2 \%$ & $45,9 \%$ & $21,3 \%$ & $6,6 \%$ & $100,0 \%$ \\
\hline \multirow{2}{*}{ Total } & 23 & 36 & 23 & 7 & 89 \\
& $25,8 \%$ & $40,4 \%$ & $25,8 \%$ & $7,9 \%$ & $100,0 \%$ \\
\hline
\end{tabular}

$p<=0.10$ 
Tabla N. ${ }^{0}$ 7. Relación entre vida familiar según el tipo de contrato laboral.

\begin{tabular}{ccccc}
\hline Vida familiar & \multicolumn{3}{c}{ Tipo de contrato } & Total \\
\hline \multirow{2}{*}{ Bajo } & Contrato SNP & Contrato eventual & Nombrado & \multirow{2}{*}{8} \\
\cline { 2 - 4 } & 6 & 1 & 1 & $100,0 \%$ \\
\multirow{2}{*}{ Medio } & $75,0 \%$ & $12,5 \%$ & $12,5 \%$ & \\
& 5 & 0 & 14 & 19 \\
\multirow{2}{*}{ Alto } & 26,3 & $0 \%$ & $73,7 \%$ & $100,0 \%$ \\
\hline \multirow{2}{*}{ Total } & $45,9 \%$ & 6 & 27 & 61 \\
& 39 & $9,8 \%$ & $44,3 \%$ & $100,0 \%$ \\
\hline
\end{tabular}

$p<=0.05$

Tabla N. ${ }^{\circ}$ 8. Relación entre vida familiar según trabaja en otra institución.

\begin{tabular}{cccc}
\hline Vida familiar & \multicolumn{2}{c}{ Trabaja en otra institución } & Total \\
\cline { 2 - 3 } Bajo & \multicolumn{1}{c}{ Sí } & No & 8 \\
& 1 & 7 & $100,0 \%$ \\
& $12,5 \%$ & $87,5 \%$ & 20 \\
\multirow{2}{*}{ Medio } & 11 & 9 & $100,0 \%$ \\
& $55,0 \%$ & $45,0 \%$ & 61 \\
\multirow{2}{*}{ Alto } & 22 & 39 & $100,0 \%$ \\
\hline \multirow{2}{*}{ Total } & $36,1 \%$ & $63,9 \%$ & 89 \\
& 34 & 55 & $100,0 \%$ \\
\hline
\end{tabular}

$p<=0.10$

Tabla N. ${ }^{0}$ 9. Grado de despersonalización según nivel de estudios.

\begin{tabular}{cccc}
\hline Despersonalización & \multicolumn{2}{c}{ Nivel de estudios } & Total \\
\hline \multirow{2}{*}{ Bajo } & Profesional & Postgrado & 62 \\
\cline { 2 - 3 } & 42 & 20 & $100,0 \%$ \\
\hline \multirow{2}{*}{ Medio } & $67,7 \%$ & $32,3 \%$ & 26 \\
& 22 & 4 & $100,0 \%$ \\
\hline \multirow{2}{*}{ Alto } & $84,6 \%$ & $15,4 \%$ & 1 \\
& 0 & 1 & $100,0 \%$ \\
\hline \multirow{2}{*}{ Total } & $0 \%$ & $100,0 \%$ & 89 \\
& 64 & 25 & $100,0 \%$ \\
\hline
\end{tabular}

$p<=0.10$ 
Tabla N. ${ }^{\circ}$ 10. Realización profesional según el tiempo de trabajo en su vida.

\begin{tabular}{|c|c|c|c|c|c|c|c|}
\hline $\begin{array}{l}\text { Realización } \\
\text { profesional }\end{array}$ & \multicolumn{6}{|c|}{ Tiempo de trabajo en su vida } & Total \\
\hline Medio & $\begin{array}{c}- \text { de } 1 \\
\text { año } \\
\end{array}$ & $\begin{array}{l}1 \text { a } 5 \\
\text { años }\end{array}$ & $\begin{array}{c}6 \text { a } 10 \\
\text { años }\end{array}$ & $\begin{array}{c}11 \text { a } 20 \\
\text { años }\end{array}$ & $\begin{array}{c}21 \text { a } 30 \\
\text { años }\end{array}$ & $\begin{array}{c}+ \text { de } 30 \\
\text { años }\end{array}$ & \multirow{2}{*}{$\begin{array}{c}18 \\
100,0 \%\end{array}$} \\
\hline меніо & $\begin{array}{c}2 \\
11,1 \%\end{array}$ & $\begin{array}{c}1 \\
5,6 \%\end{array}$ & $\begin{array}{c}10 \\
55,6 \%\end{array}$ & $\begin{array}{c}4 \\
22,2 \%\end{array}$ & $\begin{array}{c}0 \\
0 \%\end{array}$ & $\begin{array}{c}1 \\
5,6 \%\end{array}$ & \\
\hline Alto & $\begin{array}{c}3 \\
4,2 \% \\
\end{array}$ & $\begin{array}{c}16 \\
22,5 \% \\
\end{array}$ & $\begin{array}{c}16 \\
22,5 \% \\
\end{array}$ & $\begin{array}{c}26 \\
36,6 \% \\
\end{array}$ & $\begin{array}{c}8 \\
11,3 \% \\
\end{array}$ & $\begin{array}{c}2 \\
2,8 \% \\
\end{array}$ & $\begin{array}{c}71 \\
100,=\%\end{array}$ \\
\hline Total & $\begin{array}{c}5 \\
5,6 \%\end{array}$ & $\begin{array}{c}17 \\
19,1 \%\end{array}$ & $\begin{array}{c}26 \\
29,2 \%\end{array}$ & $\begin{array}{c}30 \\
33,7 \%\end{array}$ & $\begin{array}{c}8 \\
9,0 \%\end{array}$ & $\begin{array}{c}3 \\
3,4 \%\end{array}$ & $\begin{array}{c}89 \\
100,0 \%\end{array}$ \\
\hline
\end{tabular}

Tabla N. ${ }^{\circ}$ 11. Superior inmediato según antigüedad en la institución.

\begin{tabular}{|c|c|c|c|c|c|c|}
\hline $\begin{array}{c}\text { Superior } \\
\text { inmediato }\end{array}$ & \multicolumn{5}{|c|}{ Antigüedad en la institución } & Total \\
\hline & - de 1 año & 1 a 5 años & 6 a 10 años & 11 a 20 años & 21 a 30 años & \multirow[b]{2}{*}{$\begin{array}{c}18 \\
100,0 \%\end{array}$} \\
\hline Bajo & $\begin{array}{c}6 \\
33,3 \%\end{array}$ & $\begin{array}{c}2 \\
11,1 \%\end{array}$ & $\begin{array}{c}4 \\
22,2 \%\end{array}$ & $\begin{array}{c}4 \\
22,2 \%\end{array}$ & $\begin{array}{c}2 \\
11,1 \%\end{array}$ & \\
\hline Medio & $\begin{array}{c}3 \\
8,8 \%\end{array}$ & $\begin{array}{c}10 \\
29,4 \%\end{array}$ & $\begin{array}{c}5 \\
14,7 \%\end{array}$ & $\begin{array}{c}12 \\
35,3 \%\end{array}$ & $\begin{array}{c}4 \\
11,8 \%\end{array}$ & $\begin{array}{c}34 \\
100,0 \%\end{array}$ \\
\hline Alto & $\begin{array}{c}5 \\
13,5 \%\end{array}$ & $\begin{array}{c}10 \\
27,0 \%\end{array}$ & $\begin{array}{c}15 \\
40,5 \%\end{array}$ & $\begin{array}{c}6 \\
16,2 \%\end{array}$ & $\begin{array}{c}1 \\
2,7 \%\end{array}$ & $\begin{array}{c}37 \\
100,0 \%\end{array}$ \\
\hline Total & $\begin{array}{c}14 \\
15,7 \%\end{array}$ & $\begin{array}{c}22 \\
24,7 \%\end{array}$ & $\begin{array}{c}24 \\
27,0 \%\end{array}$ & $\begin{array}{c}22 \\
24,7 \%\end{array}$ & $\begin{array}{c}7 \\
7,9 \%\end{array}$ & $\begin{array}{c}89 \\
100,0 \%\end{array}$ \\
\hline
\end{tabular}

Tabla N. ${ }^{\circ}$ 12. Superior inmediato según antigüedad en el puesto.

\begin{tabular}{|c|c|c|c|c|c|}
\hline Superior inmediato & & Antigüed: & I en el puesto & & Total \\
\hline \multirow[b]{2}{*}{ Bajo } & - de 1 año & 1 a 5 años & 6 a 10 años & 11 a 20 años & \multirow{2}{*}{$\begin{array}{c}18 \\
100,0 \%\end{array}$} \\
\hline & $\begin{array}{c}6 \\
33,3 \%\end{array}$ & $\begin{array}{c}2 \\
11,1 \%\end{array}$ & $\begin{array}{c}5 \\
27,8 \%\end{array}$ & $\begin{array}{c}5 \\
27,8 \%\end{array}$ & \\
\hline Medio & $\begin{array}{c}8 \\
23,5 \%\end{array}$ & $\begin{array}{c}13 \\
38,2 \%\end{array}$ & $\begin{array}{c}5 \\
14,7 \%\end{array}$ & $\begin{array}{c}8 \\
23,5 \%\end{array}$ & $\begin{array}{c}34 \\
100,0 \%\end{array}$ \\
\hline Alto & $\begin{array}{c}6 \\
16,7 \%\end{array}$ & $\begin{array}{c}16 \\
44,4 \%\end{array}$ & $\begin{array}{c}13 \\
36,1 \%\end{array}$ & $\begin{array}{c}1 \\
2,8 \%\end{array}$ & $\begin{array}{c}36 \\
100,0 \%\end{array}$ \\
\hline Total & $\begin{array}{c}20 \\
22,7 \%\end{array}$ & $\begin{array}{c}31 \\
35,2 \%\end{array}$ & $\begin{array}{c}23 \\
26,1 \%\end{array}$ & $\begin{array}{c}14 \\
15,9 \%\end{array}$ & $\begin{array}{c}88 \\
100,0 \%\end{array}$ \\
\hline
\end{tabular}


Tabla N. ${ }^{\circ}$ 13. Compromiso afectivo con la profesión según antigüedad en la institución.

\begin{tabular}{ccccccc}
\hline \multirow{2}{*}{$\begin{array}{c}\text { Compromiso } \\
\text { afectivo }\end{array}$} & $\begin{array}{c}\text { - de } \mathbf{1} \\
\text { año }\end{array}$ & $\begin{array}{c}\mathbf{1} \text { a 5 } \\
\text { años }\end{array}$ & $\begin{array}{c}\mathbf{6} \text { a 10 } \\
\text { años }\end{array}$ & $\begin{array}{c}\mathbf{1 1} \text { a 20 } \\
\text { años }\end{array}$ & $\begin{array}{c}\mathbf{2 1} \text { a 30 } \\
\text { años }\end{array}$ & TOTAL \\
\cline { 2 - 5 } Bajo & 1 & 0 & 1 & 1 & 0 & \\
& $33,3 \%$ & $0 \%$ & $33,3 \%$ & $33,3 \%$ & $0 \%$ & $100,0 \%$ \\
\hline \multirow{2}{*}{ Medio } & 4 & 15 & 13 & 13 & 7 & 52 \\
& $7,7 \%$ & $28,8 \%$ & $25,0 \%$ & $25,0 \%$ & $13,5 \%$ & $100,0 \%$ \\
\hline \multirow{2}{*}{ Alto } & 9 & 7 & 10 & 8 & 0 & 34 \\
& $26,5 \%$ & $20,6 \%$ & $29,4 \%$ & $23,5 \%$ & $0 \%$ & $100,0 \%$ \\
\hline \multirow{2}{*}{ Total } & 14 & 22 & 24 & 22 & 7 & 89 \\
& $15,7 \%$ & $24,7 \%$ & $27,0 \%$ & $24,7 \%$ & $7,9 \%$ & $100,0 \%$ \\
\hline
\end{tabular}

$P<=0.10$

Tabla N. ${ }^{0}$ 14. Relación entre estrés laboral e ingreso neto mensual.

\begin{tabular}{ccccc}
\hline \multirow{2}{*}{ Estrés laboral } & \multicolumn{3}{c}{ Ingreso neto mensual en soles $\mathbf{( S / . * )}$} & \multirow{2}{*}{ Total } \\
\cline { 2 - 4 } & Hasta $\mathbf{1 0 0 0}$ & $\mathbf{1 0 0 1}$ a 3000 & $\mathbf{3 0 0 1}$ a 5000 & \\
\hline \multirow{2}{*}{ Bajo } & 24 & 24 & 4 & 52 \\
& $46,2 \%$ & $46,2 \%$ & 7,7 & $100,0 \%$ \\
\hline \multirow{2}{*}{ Medio } & 15 & 12 & 2 & 29 \\
& $51,7 \%$ & $41,4 \%$ & $6,9 \%$ & $100,0 \%$ \\
\hline \multirow{2}{*}{ Alto } & 0 & 8 & 0 & 8 \\
& $0 \%$ & $100,0 \%$ & $0 \%$ & $100,0 \%$ \\
\hline \multirow{2}{*}{ Total } & 39 & 44 & 6 & 89 \\
& $43,8 \%$ & $49,4 \%$ & $6,7 \%$ & $100,0 \%$ \\
\hline
\end{tabular}

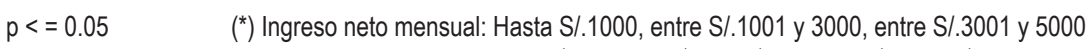
Equivalencia en dólares: Hasta $\$ 334$, entre $\$ 333$ y $\$ 999$, entre $\$ 1000$ y $\$ 1667$

Tabla N. ${ }^{0}$ 15. Relación entre satisfacción salarial e ingreso neto mensual.

\begin{tabular}{ccccc}
\hline \multirow{2}{*}{$\begin{array}{c}\text { Satisfacción } \\
\text { salarial }\end{array}$} & \multicolumn{2}{c}{ Ingreso neto mensual en soles (S/.) } & \multirow{2}{*}{ Total } \\
\cline { 2 - 4 } Hasta $\mathbf{1 0 0 0}$ & $\mathbf{1 0 0 1}$ a 3000 & $\mathbf{3 0 0 1}$ a 5000 & \\
\hline \multirow{2}{*}{ Bajo } & 23 & 20 & 4 & 47 \\
& $48,9 \%$ & $42,6 \%$ & $8,5 \%$ & $100,0 \%$ \\
\hline \multirow{2}{*}{ Medio } & 15 & 14 & 1 & 30 \\
& $50,0 \%$ & $46,7 \%$ & $3,3 \%$ & $100,0 \%$ \\
\hline \multirow{2}{*}{ Alto } & 1 & 10 & 1 & 12 \\
& $8,3 \%$ & $83,3 \%$ & $8,3 \%$ & $100,0 \%$ \\
\hline \multirow{2}{*}{ Total } & 39 & 44 & 6 & 89 \\
& $43,8 \%$ & $49,4 \%$ & $6,7 \%$ & $100,0 \%$ \\
\hline$p<=.10$ & $(*)$ Ingreso neto mensual: Hasta S/. 1000, entre S/. 1001 y 3000, entre S/. 3001 y 5000 \\
& Equivalencia en dólares: Hasta $\$ 333$, entre $\$ 334$ y $\$ 999$, entre $\$ 1000$ y $\$ 1667$
\end{tabular}


Tabla N. ${ }^{\circ}$ 16. Compromiso afectivo con la profesión según ingreso neto mensual.

\begin{tabular}{ccccc}
\hline \multirow{2}{*}{$\begin{array}{c}\text { Compromiso } \\
\text { afectivo }\end{array}$} & \multicolumn{3}{c}{ Ingreso neto mensual* } & \multirow{2}{*}{ TOTAL } \\
\cline { 2 - 4 } Hajta 1000 & $\mathbf{1 0 0 1}$ a 3000 & $\mathbf{3 0 0 1}$ a 5000 & \\
\hline \multirow{2}{*}{ Bajo } & 0 & 3 & 0 & 3 \\
& $0 \%$ & $100.0 \%$ & $0 \%$ & $100,0 \%$ \\
\hline \multirow{2}{*}{ Medio } & 21 & 25 & 6 & 52 \\
& $40,4 \%$ & $48,1 \%$ & $11,5 \%$ & $100,0 \%$ \\
\hline \multirow{2}{*}{ Tlto } & 18 & 16 & 0 & 34 \\
& $52,9 \%$ & $47,1 \%$ & $0 \%$ & $100,0 \%$ \\
\hline
\end{tabular}

$p<=0.05 \quad\left({ }^{*}\right)$ Ingreso neto mensual: Hasta S/.1000, entre S/. 1001 y 3000 , entre S/. 3001 y 5000

Equivalencia en dólares: Hasta \$333, entre \$ 334 y \$ 999, entre \$ 1000 y \$ 1667

Tabla N. ${ }^{0}$ 17. Búsqueda de otro empleo según ingreso neto mensual.

\begin{tabular}{ccccc}
\hline \multirow{2}{*}{$\begin{array}{c}\text { Búsqueda de } \\
\text { otro empleo }\end{array}$} & \multicolumn{2}{c}{ Ingreso neto mensual en soles* (S/.) } & \multirow{2}{*}{ Total } \\
\cline { 2 - 4 } & Hasta $\mathbf{1 0 0 0}$ & $\mathbf{1 0 0 1} \mathbf{a} \mathbf{3 0 0 0}$ & $\mathbf{3 0 0 1}$ a 5000 & \\
\hline \multirow{2}{*}{ Bajo } & 22 & 32 & 1 & 55 \\
& $40,0 \%$ & $58,2 \%$ & $1,8 \%$ & $100,0 \%$ \\
\hline \multirow{2}{*}{ Medio } & 12 & 8 & 3 & 23 \\
& $52,2 \%$ & $34,8 \%$ & $13,0 \%$ & $100,0 \%$ \\
\hline \multirow{2}{*}{ Alto } & 5 & 4 & 2 & 11 \\
& $45,5 \%$ & $36,4 \%$ & $18,2 \%$ & $100,0 \%$ \\
\hline \multirow{2}{*}{ Total } & 39 & 44 & 6 & 89 \\
& $43,8 \%$ & $49,4 \%$ & $6,7 \%$ & $100,0 \%$ \\
\hline
\end{tabular}

$p<=.10 \quad\left(^{*}\right)$ Ingreso neto mensual: Hasta S/.1000, entre S/. 1001 y 3000 , entre S/. 3001 y 5000

Equivalencia en dólares: Hasta \$333, entre \$334 y \$999, entre \$1000 y \$1667 